\title{
Inteligencia emocional y creencias irracionales en estudiantes universitarios de fisioterapia
}

\author{
Emotional intelligence and irrational beliefs in physiotherapy university students
}

Inteligência emocional e crenças irracionais em estudantes universitários de fisioterapia

Karen Arráez

karentemporalr@gmail.com

https://orcid.org/0000-0003-4711-7444

Universidad Arturo Michelena, Valencia, Venezuela
María Castro

mariacastrotr3@gmail.com

https://orcid.org/0000-0002-7999-0099

Universidad Arturo Michelena, Valencia, Venezuela
RESUMEN

El presente estudio tuvo como objetivo determinar la relación entre la Inteligencia Emocional y las creencias Irracionales en estudiantes universitarios de Fisioterapia con edades comprendidas entre 18 y 28 de edad pertenecientes a familias monoparentales aplicado en las instalaciones de la Universidad Arturo Michelena. El criterio metodológico la investigación fue un diseño de campo no experimental, tipo transversal con nivel correlacional y modalidad cuantitativa.. Para el evaluar los resultados se aplicó el coeficiente de Pearson, el cual permitió demostrar las correlaciones significativas, reflejadas a través de gráficos estadísticos para su comparación, concluyendo la existencia de la correlación entre la Inteligencia Emocional y las Creencias Irracionales.

Palabras clave: Inteligencia emocional; creencias irracionales; estudiantes universitarios; familia monoparentales
ABSTRACT

The present study aims to determine the relationship between Emotional Intelligence and Irrational beliefs in university students of Physiotherapy with ages between 18 and 28 of age belonging to single-parent families applied in the facilities of the University of Arturo Michelena. The methodological criteria of the investigation were a non-experimental field design, transversal type with correlational level and quantitative mode. To evaluate the results, the Pearson coefficient was applied, which allowed to demonstrate the significant correlations, reflected through statistical graphs for its comparison, concluding the existence of a correlation between Emotional Intelligence and Irrational creencias.

Palabras clave: Emotional intelligence; irrational beliefs; university students; single parent family
O presente estudio tuvo como objetivo determinar a relación entre la Inteligencia Emocional y las creencias irracionales en estudiantes universitarios de Fisioterapia con edades comprendidas entre 18 y 28 de edad pertenecientes a familias monoparentales at the instalaciones de la Universidad Arturo Michelena. El criterio metodológico la investigación fue un diseño de campo no experimental, tipo transversal con nivel correlacional y modalidad cuantitativa. Para el avaliar los resultados se aplicó el coeficiente de Pearson, el cual permitió demostrar las correlaciones, reflejadas a través de gráficos estaduais para su comparación, concluyendo la existencia de la correlación entre la Inteligencia Emocional y las Creencias Irracionales.

Palavras-chave: Inteligência Emocional; Crenças irracionais; estudantes universitarios; família monoparental 


\section{INTRODUCCIÓN}

La inteligencia humana permite al hombre desarrollar el potencial para administrar sus capacidades de aprender, razonar, adaptarse al entorno, entre otras. Es por ello que, la American Psychological Association APA 1996, citado en Ceballos, 2015, lo expuso así:

Los individuos difieren los unos de los otros en habilidad de comprender ideas complejas, de adaptarse eficazmente al entorno, así como el de aprender de la experiencia, en encontrar varias formas de razonar, de superar obstáculos mediante la reflexión.... El concepto de "inteligencia" es una tentativa de aclarar y organizar este conjunto complejo de fenómenos (s.n.).

Dentro de esta perspectiva, los seres humanos están dotados de sentidos que les permiten la relación con el ambiente que los rodea, admitiendo la percepción de los estímulos que junto a la inteligencia desarrollada incentivará la evolución del hombre, siendo cada estímulo interpretado de acuerdo con la estructura de creencias y valores con la que la persona ha sido educada desde niño. Sin embargo, estas creencias pueden no estar apegadas a la realidad, pueden ser ideas irracionales que desencadenen una respuesta desadaptativa.

En este sentido, una creencia irracional es cualquier pensamiento que tomado como verdadero, patrocina una o más afirmaciones, indicando que todo debe ser concebido de una forma establecida o que el individuo que manifieste dicha irracionalidad debe acoplarse a un modelo preciso de comportamiento, induciendo consecuencias desacertadas y autodestructivas.

Por tanto, el ser humano actúa con racionalidad, por lo que es preciso el uso de la inteligencia emocional para logar el equilibrio entre las ideas y acciones, vinculadas con un conjunto de habilidades, que se basan en la capacidad de reconocer los sentimientos propios y de los demás, sirviendo de guía al pensamiento. A través de la inteligencia emocional, el individuo conquista relaciones sanas con las personas en los diferentes ámbitos en los que se desenvuelve, generando confianza en sí mismo y actitud mental positiva.

Es oportuno destacar para problematizar que la familia es la base fundamental de la sociedad, la cual representa los elementos necesarios en la formación del individuo, como lo expresa Valladares (2008):
La familia es una institución formada por sistemas individuales que interactúan y que constituyen a su vez un sistema abierto. Está formada por individuos, es también parte del sistema social y responde a su cultura y tradiciones, desarrollo económico, convicciones, concepciones éticas, morales, políticas y religiosas (p.4).

Por consiguiente, el ser humano precisa vivir en familia, a este propósito comenta Valladares (2008): “La necesidad de vivir en familia que tiene el ser humano se acrecienta ante el carácter eminentemente psicológico que tiene la relación niño- adulto" (p.5), lo cual permite el desarrollo del individuo y de la familia en el contexto social. Por otra parte, en la Declaración de UNICEF (2003) en América Latina y Caribe se asegura que: "La familia es y seguirá siendo la red básica de relaciones sociales y elemento fundamental de cohesión social; es irremplazable en la funciones de socialización, del desarrollo de la afectividad de transmisión de valores..." (s.n.). Lo que pone de manifiesto que la familia es insustituible, otorgando al individuo el clima familiar propicio para su desarrollo. 
En relación a lo antes expuesto, la familia según Valladares (2008):

El estudio de la familia como grupo social constituye una necesidad para el profesional de la salud, por ser este el grupo que más influye en la formación de la personalidad, en ella se adquieren las creencias, los temores y criterios de riesgos que condicionan las actitudes de la persona (p.12).

Ahora bien, dentro del contexto familiar es importante el engranaje de todos sus miembros, formando la llamada familia nuclear, compuesta por papá, mamá e hijos. Sin embargo, esto ha ido cambiando como así lo señala UNICEF (2003): "El conjunto de transformaciones que ha experimentado la familia en el mundo occidental constituye una de las manifestaciones más importantes del cambio social contemporáneo". (p.7). Ocasionando que estos cambios invadieran espacios de la tradicional familia nuclear como así lo expresa UNICEF (2003):

En pocas décadas, el modelo de la familia afianzado en la inmediata posguerra, ampliamente difundido bajo el rótulo de la "familia nuclear", fue cediendo espacio a una creciente diversidad de formas y estilos de vida familiares...Al lado de la familia nuclear "tradicional", comenzaron a cobrar relevancia numérica y social, las familias monoparentales y las familias "reconstituidas o ensambladas" (p.8).

En virtud a esto, las familias monoparentales han ido creciendo, debido a la ausencia de la madre o el padre, por lo que, esta investigación hará énfasis en las consecuencias de la misma, como medio condicionante en la inteligencia emocional y las creencias irracionales. Basado en lo anterior, el gobierno de Chile realizó la encuesta de Caracterización Socioeconómica Nacional
(CASEN, 2014) indicando que en países como México, EE.UU, Colombia, Chile, entre otros., arrojó que en el año 2013, el 33,6\% de los hijos viven solo con la madre o el padre, ubicándolos dentro del contexto de familia monoparental, lo que acarrea problemas de diferentes índole en el entorno social y psicológico en el desarrollo del individuo.

Cabe agregar que, según el Censo Nacional de Población y Vivienda (INE 2011) el 39,3\% de las mujeres son jefes de hogar. Sin embargo, los analistas de estadísticas sociales coinciden que estos datos encubren un sub-registro, el cual logra estar determinado por la propia seguridad del hogar, debido a que en los sectores populares y no populares, la mujer es renuente a proporcionar información a un desconocido, alterando así la veracidad de los datos. Ahora bien, la ausencia de alguno de los progenitores puede ocasionar en el sujeto, trastornos o cambios referidos a perturbaciones emocionales, condicionadas por las creencias o ideas irracionales. En el caso de los estudiantes universitarios, esto repercute en su conducta, lo que debe ser considerado para el estudio.

Según Ellis (1980), señala que:

Las creencias irracionales, constituyen distorsiones de la realidad y configuran una personalidad con demandas excesivas, a través de un proceso de etiquetar a las personas, y que actúan como bloqueadores y por lo tanto, son obstáculos para el logro adecuado de las interacciones interpersonales, y que en su expresión más inmediata afectan la convivencia y la salud mental (p.15).

Se debe añadir que la inteligencia emocional para Goleman (1985): "se enlaza con las habilidades basadas en la capacidad del individuo de reconocer los sentimientos propios y ajenos, sirviendo de guía alpensamiento"(p.13), definiéndola Goleman(1985) como: "la capacidad de reconocer nuestros propios sentimientos y los de los demás, de motivarnos y 
de manejar adecuadamente las relaciones" (p.45), contribuyendo a la consolidación del desarrollo del individuo. De allí que, el estudiante universitario afronta creencias irracionales que le impiden un desenvolvimiento satisfactorio en los diferentes ámbitos en los que se relaciona, ocasionándole serios problemas de adaptabilidad y desempeño, algunas de estas creencias pueden tener sus cimientos en el entorno familiar, perturbando el comportamiento del individuo.

Es importante realzar que, el control de estas creencias permitirá al individuo una actuación equilibrada a través de estrategias inteligentes que le suministren al estudiante el desarrollo de conductas racionales. A este propósito, se tomó como población para el caso estudio a estudiantes de Fisioterapia cursante del $2^{\circ}$ al $9^{\circ}$ cuatrimestre de la Universidad Arturo Michelena, comprendidos entre los 18 a 28 años de edad pertenecientes a familias monoparentales.

\section{Interrogantes de la investigación}

- ¿Qué nivel de inteligencia emocional presentan los estudiantes de Fisioterapia entre 18 y 28 años de edad pertenecientes a familias monoparentales de la Universidad Arturo Michelena?

- ¿Existirán creencias irracionales en los estudiantes de Fisioterapia entre 18 y 28 años de edad pertenecientes a familias monoparentales de la Universidad Arturo Michelena?

- ¿Existirá relación entre inteligencia emocional y creencias irracionales en los estudiantes de Fisioterapia entre 18 y 28 años de edad pertenecientes a familias monoparentales de la Universidad Arturo Michelena?

Se enuncia así el objetivo de investigación:

- Determinar la relación entre la inteligencia emocional y creencias irracionales en estudiantes universitarios de Fisioterapia entre
18 y 28 de edad pertenecientes a familias monoparentales de la Universidad Arturo Michelena.

Ante lo expuesto se justifica el presente estudio, entre varios aspectos, porque la vida universitaria como cualquier ámbito de la existencia, está inmersa en logros, frustraciones, nuevas exigencias y experiencias. Igualmente, el aprendizaje de las habilidades que el universitario demanda, ajustándose y desenvolviéndose de manera apropiada en su dominio personal y profesional, requiere de un historial de experiencias anteriores, las cuales le permitan establecer estrategias para enfrentar las vicisitudes.

Por ello, la presente investigación basada en inteligencia emocional y creencias irracionales en los estudiantes universitarios de fisioterapia entre 18 y 28 años de edad pertenecientes a familias monoparentales, pretendió establecer la relación entre estas variables dentro del contexto educativo y social en el que se desarrolló la investigación.

De igual manera, bajo los esquemas establecidos, contribuirá a futuras investigaciones, no solamente en la Universidad Arturo Michelena, sino en el ámbito nacional, sirviendo de utilidad en el área de salud mental.

Por consiguiente, este estudio pretendió suministrar ideas que permitan mejorar los índices de creencias irracionales en el estudiante universitario, a través de la inteligencia emocional, apuntando a la disminución de conductas inadecuadas que generan problemas a nivel emocional como en su desarrollo psicosocial y familiar.

Paraestecometidodel proceso deinvestigación, se consideraron un conjunto de estudios previos que amplían los referentes problemáticos

\section{Antecedentes}

Huerta, J. (2012) en su investigación titulada: "Inteligencia e ideas irracionales en estudiantes 
universitarios" Participaron 28 alumnos de la Universidad Tecnológica de Huejotzingo (UTH) en Puebla. México. Se aplicó la escala de pensamientos irracionales de Albert Ellis y la prueba de inteligencia WAIS-III. Los resultados se analizaron por medio de correlaciones de Spearman entre las escalas de Ellis y el WAISIII. Se obtuvo 294 correlaciones, de las cuales sólo 5 apoyaron significativamente la hipótesis con valores $r$ entre -0.42 y -0.69 . En conclusión, se encontró poca evidencia de que a mayor inteligencia menos ideas irracionales. Por ello, este estudio contribuye con datos significativos, debido a que el trabajo se enfoca en el mismo contexto de la presente investigación con dos variables en común como son ideas irracionales e inteligencia aplicado en universitarios, lo cual es de utilidad para el marco teórico, y como referencia para la futura observación y análisis de resultados.

Mazariegos, M. (2013) realizó el estudio titulado: "Relación entre las ideas irracionales y la comunicación disfuncional en la familia". Estudio realizado con adolescentes de 14 a 17 años de INEB adscrito a la Escuela Normal de Maestras de Educación para el hogar de Quetzaltenango en Guatemala. El objetivo de la presente investigación fue determinar la influencia que tienen las ideas irracionales en los adolescentes como causa de la comunicación disfuncional en la familia. El tipo de investigación realizada fue descriptiva, tomándose como estudio un grupo de control. Para poder obtener resultados se utilizó el Test Inventario de Ideas Irracionales y el cuestionario de funcionamiento familiar. Se evidenció la presencia de ideas irracionales en los adolescentes evaluados, arrojando un $50 \%$ dentro del área nublada, que indica que exageran las consecuencias negativas de un hecho o acontecimiento.

Por cuanto, dentro del rango brumoso se encuentra el $42 \%$, que indica un alto grado de ideas irracionales, provocando una inadaptación al medio en donde se desenvuelven, produce emociones negativas intensas y duraderas. Con un $4 \%$ se encuentran los adolescentes dentro del rango turbio que significa que deben tener ayuda psicológica urgente, pues la cantidad de ideas irracionales que manejan afectan su vida en todos los ámbitos en que se desenvuelven, debido a que pueden encontrarse en una fuerte depresión a causa de las dosis altas de auto castigo y verse como merecedoras de recibirlo. Este trabajo aporta al presente estudio la eficacia de la aplicación del Test de Ideas Irracionales permitiendo analizar las mismas dentro del ámbito familiar.

Rosal, I., Dávila, M., Sánchez, S., Bermejo, M. (2016) llevaron a cabo una investigación en la Universidad de Extremadura España, titulado "La Inteligencia Emocional en estudiantes universitarios: Diferencias entre el Grado de Maestro en Educación primaria y los Grados en Ciencias". Mediante una metodología cuantitativa, y más concretamente a partir de un diseño transversal, evaluando el nivel de inteligencia emocional en una muestra constituida por 358 estudiantes del Grado de Maestro en Educación y Grados en Ciencias de la Universidad de Extremadura, con el test de autoinforme TMMS-24 de FernándezBerrocal, Extremera y Ramos (2004).

Deallíque los resultados obtenidos reconocieron que existen diferencias estadísticamente significativas en el nivel reparación emocional según el sexo, en cada una de las dimensiones que conforman la inteligencia emocional, según el grado en el que se encuentran matriculados los estudiantes universitarios.

En cuanto a las investigaciones nacionales se inicia con Pereira, (2014) presenta un trabajo titulado: "Estudio de la Inteligencia Emocional en el manejo de conflictos laborales de los empleados de la Oficina de Personal del Gobierno Bolivariano de Carabobo". Universidad de Carabobo. Esta investigación se desarrolló con el objetivo principal 
de estudiar la inteligencia emocional en el manejo de conflictos laborales del personal administrativo específicamente del Departamento de Registro y Control de la Gobernación Bolivariana de Carabobo.

Se tomó como antecedente esta investigación, ya que tuvo como finalidad evaluar los niveles de inteligencia emocionalenestudiantesuniversitarios coincidiendo con la investigación actual en cuanto a los niveles de la misma, aplicándose a una muestra sustraída de la institución educativa.

Seguidamente, se establecieron las causas que generan conflictos en el personal administrativo y el manejo de las emociones, en cuanto a la conducción de conflictos, asimismo se determinó una relación entre la inteligencia emocional y el manejo de conflictos en el referido departamento. En virtud a la metodología utilizada se enmarcó en una investigación de campo, descriptiva, abarcando una población de 15 empleados, tomándose como muestra el $100 \%$ de ellos; los cuales accedieron a la aplicación de un cuestionario contentivo de 13 ítems respectivamente, validado por expertos cuyo resultados se mostraron en cuadros de frecuencia y porcentajes, agrupados finalmente en gráficos sectoriales.

Por consiguiente, se concluyó que los empleados administrativos del Departamento de registro y Control adscrito a la Gobernación Bolivariana de Carabobo no presentan condiciones adecuadas en el manejo de conflictos laborales y no poseen control de sus emociones, ante las situaciones y adversidades que diariamente afronta dicha oficina; cabe destacar que existen múltiples elementos organizacionales, que causan malestar en el personal, tales como la insatisfacción salarial, falta de comunicación, vocación de servicio, escasa valoración del personal por las labores ejecutadas y la falta de capacitación del personal referente al manejo de las emociones, generando como consecuencia conflictos laborales. El aporte de este estudio investigativo se refiere a la importancia del manejo de las emociones para la solución de conflictos, lo cual se puede compartir con las ideas irracionales que se generan en la vida estudiantil.

Hernández y Moreno (2015) realizaron una investigación en la Universidad Arturo Michelena titulada: "La ausencia de la figura paterna y su relación con la estructura de personalidad y la autoimagen de los adolescentes entre 18 a 20 años de edad", teniendo como objetivo general observar si la ausencia paterna de los adolescentes de 18- 20 años de edad, estudiantes del primer cuatrimestre de la Escuela de Psicología de la Universidad Arturo Michelena, se correlacionan o no con la estructura de la personalidad y su autoimagen. La muestra estuvo conformada por 59 alumnos del primer cuatrimestre y de ambos sexo, los cuales se dividieron en 30 alumnos con ausencia paterna y 29 con presencia de la figura paterna, siendo una investigación cuali-cuantitativa, de campo no experimental. Esta investigación hace su aporte a través de la incidencia que fomenta la figura paterna y la ausencia de la misma en el comportamiento del individuo.

Segura (2016) realizó un estudio titulado: Inteligencia Emocional en estudiantes venezolanos de educación media y universitaria. La presente investigación tiene por objetivo diagnosticar las habilidades emocionales de estudiantes venezolanos de educación media y universitaria, para determinar relaciones entre habilidades emocionales y los efectos del género, contexto geográfico y edad en dichas habilidades emocionales. El nivel de investigación fue exploratorio, descriptivo y correlacional. Con una población de estudiantes del $3^{\circ}, 4^{\circ}, 5^{\circ}$ año de educación media y estudiantes de los primeros semestres universitarios de las escuelas de fisioterapia e informática.

En este sentido, la muestra estuvo conformada por 441 estudiantes del recinto de educación media: Instituto Fernando de Magallanes. José Vicente de 
Unda y San Martin de Porra conjuntamente con el Colegio Universitario Cecilio Acosta. El instrumento de recogida de datos fue un cuestionario de emociones basado sobre el modelo de Goleman (1998). Para evaluar las habilidades emocionales de empatía, motivación, competencia social, autoconciencia del primer nivel, autoconciencia del segundo nivel y autocontrol, el cuestionario fue organizado en cinco ítems por habilidad emocional para un total de 30 ítems, los cuales se responden sobre una escala del tipo Likert de 5 puntos. Todos los ítems están distribuidos aleatoriamente.

Por tal motivo, los resultados revelan la existencia de diferencias estadísticas significativas entre las habilidades emocionales de los estudiantes por género, existiendo relación entre las habilidades emocionales y las habilidades de autocontrol, siendo las competencias sociales las menos favorecidas en el estudio. Es de hacer notar que el aporte entregado por el estudio a la presente investigación, radica en la importancia que representa la inteligencia emocional en la búsqueda del control de las emociones.

\section{Marco teórico}

\section{Teoría psicosocial de Erick Erickson}

El ser humano transita por etapas a lo largo de su Desarrollo Evolutivo, lo que lleva a construir la propia identidad del individuo; cada una contiene características que hacen la diferencia, Erick Erickson, (citado en Boeree, 2006). Postuló ocho etapas del desarrollo del ciclo de vida de los individuos, las cuales se describirán a continuación, en ella se determinan funciones psicosociales, que se irán superando a medida que el individuo se vaya desarrollando de forma positiva.

- Confianza básica vs Desconfianza (desde el nacimiento hasta aproximadamente los 18 meses). Él bebé recibe el calor del cuerpo de la madre y sus cuidados amorosos. Se desarrolla el vínculo que será la base de futuras relaciones con otras personas importantes; es receptivo a estímulos ambientales, y las experiencias más tempranas son las de frustración que proveen aceptación, seguridad y satisfacción emocional. El punto crucial o la crisis fundamental en el primer año de vida según Erickson (citado en Abarca, 2007) es el desarrollo de la confianza que se logra por medio de las conductas, actitudes, cuidado y amor que se le prodiga al niño. Pero si no se dan esas condiciones, el niño es sentido como un estorbo o una incomodidad que hay que acarrear, entonces aparece la desconfianza.

- Autonomía vs Vergüenza y Duda (desde los 18 meses hasta los 3 años aproximadamente). Etapa que se encuentra relacionada con al desarrollo muscular y de control de las eliminaciones del cuerpo, donde él bebé pasa por momentos de vergüenza y duda. Se plantea en esta etapa el dilema entre su control externo y sus necesidades internas, debe controlar sus intestinos y aprender a discriminar cuando evacuar o cuando retener, expresando de esta forma el control que el niño tiene de su autonomía como sujeto. El niño va construyendo su propia estructura de personalidad en interacción con su ambiente, llevándolo a desarrollar confianza y por tanto seguridad o autonomía o por el contrario, la vergüenza ante sí mismo y los demás.

- Iniciativa vs Culpa (desde los 3 años hasta los 5 años aproximadamente). De acuerdo con Erickson (citado en Abarca, 2007) el niño se caracteriza en esta etapa por tener una percepción más completa de su cuerpo, por su gran actividad física y por su capacidad de tomar iniciativas. En ocasiones pueden ser intolerantes con aquellos que les indican cómo actuar, pero que no cumplen con lo que dicen, tal es el caso de los adultos que no siguen las normas que dicen valorar. El reto mayor de esta etapa, es el realizar tareas y metas, dando control mental y locomotor. Se dan celos por atención de la madre y temor a la castración. Se presentan en los juegos simbolismos sobre 
fantasías sexuales que deben ser guiadas por los padres, ya que por ellos presentan muchas veces sentimientos de culpa y temores.

- Laboriosidad vs inferioridad (desde los 5 años hasta los 13 años aproximadamente). En esta etapa se inicia la escolaridad, dejan a un lado fantasías o dudas sobre su sexualidad, si las dudas fueron aclaradas por sus padres o personas importantes, llegaran a esta etapa con gran orientación. No tratan de igualarse con los padres, por el contrario, aparecen nuevos interesas como son las amistades dentro de la escuela. Esta etapa gira en torno a la curiosidad intelectual y a la ejecución es por tanto que la institución educativa debe velar por el establecimiento del sentimiento de laboriosidad.

\section{Inteligencia emocional}

Inteligencia emocional es una destreza que permite conocer y manejar las emociones, de forma de interpretar los sentimientos de los demás y como eso afecta el desenvolvimiento del individuo. Fueron Salovey y Mayer, (1997) que iniciaron los estudios en base a la Inteligencia Emocional definiéndola así:

Habilidadparapercibir,valoraryexpresar emociones con exactitud, la habilidad para acceder $y / o$ generar sentimientos que faciliten el pensamiento; la habilidad para comprender emociones y el conocimiento emocional y la habilidad para regular las emociones promoviendo un crecimiento emocional e intelectual (p.27).

Por su parte, Goleman (1995) explica que:

La inteligencia emocional, incluye el autodominio, el celo, la persistencia y la capacidad de motivarse uno mismo; Los adolescentes deben saber que la inteligencia emocional, tiene relación con los sentimientos, el carácter o los instintos morales, son la base de las emociones, que contrae una acción de impulsos como es la ira, miedo, cambios biológicos, amor, expresión de disgusto, tristeza (p.29).

Entre tanto Cherniss y Goleman (2005) comentan que: "la inteligencia emocional es tan importante en lo que concierne a la crianza del día a día a crear paz armonía en una casa debe ser realista" (p.17). Es necesario la integración del grupo familiar para lograr la armonía y la resolución de los conflictos de manera racional, evitando así la confrontación de criterios que alteren las emociones del grupo.

\section{MÉTODO}

El presente estudio fue una investigación de campo no experimental, transversal En virtud a lo señalado, realizó la recolección de datos en la Universidad Arturo Michelena, tomando en consideración la no manipulación de las variables en estudio permitiendo resultados acorde a la posible correlación existente.

\section{Sistema de variables}

Variables relacionadas

- Inteligencia emocional

- Creencias irracionales

- Variables de control

- Edad: entre los 18 y 28 años

- Cursantes $2^{\circ}$ al $9^{\circ}$ semestre de Fisioterapia en la Universidad Arturo Michelena

- Familias monoparentales de 0 meses a 13 años de edad

Variables intervinientes

- Sexo

- Dinámica familiar

- Rasgos de personalidad

- Situación país 
En cuanto a la población, la misma estuvo conformada por estudiantes pertenecientes del 2do al 9no cuatrimestre de Fisioterapia, dando un total de 870 estudiantes.

\section{RESULTADOS}

Se describen los resultados obtenidos de la investigación orientada a determinar la relación entre inteligencia emocional de la muestra de 90 estudiantes de Fisioterapia entre 18 y 28 años de edad de la Universidad Arturo Michelena pertenecientes a familias monoparentales.

Evaluación de los niveles de inteligencia emocional en los estudiantes Fisioterapia con edades comprendidas entre 18 y 28 años de edad de la Universidad Arturo Michelena pertenecientes a familias monoparentales.
Los niveles de inteligencia emocional en la muestra de estudiantes seleccionada fueron obtenidos a través de la aplicación, tabulación y análisis del Trait Meta- Mood Scale 24, adaptación de Fernández Berrocal, Extremera y Ramos (2004), obteniéndose las tablas de puntuaciones generales, para el nivel de inteligencia considerando niveles alta, adecuada y poca y también para cada una de las tres dimensiones consideradas en la adaptación para percepción emocional, comprensión emocional y regulación emocional, tanto en términos de la distribución porcentual de sus niveles, como de las puntuaciones medias obtenidas.

Tabla 1. Puntuaciones obtenidas en Inteligencia emocional.

\begin{tabular}{ccccccc}
\hline $\begin{array}{c}\text { No. } \\
\text { Estudiantes }\end{array}$ & Edad & Sexo & Trimestre & Familia tipo & $\begin{array}{c}\text { Puntaje } \\
\text { Total }\end{array}$ & $\begin{array}{c}\text { Nivel de Inteligencia } \\
\text { Emocional }\end{array}$ \\
\hline 1 & 18 & $F$ & 3ro & M(Papá 1 año) & $\mathbf{8 5 . 0}$ & IE Adecuada \\
2 & 21 & $F$ & 3ro & M(Papá 6 años) & $\mathbf{8 3 . 0}$ & IE Adecuada \\
3 & 21 & M & 3ro & M(Papá 13 años) & $\mathbf{9 3 . 0}$ & IE Adecuada \\
4 & 21 & $F$ & $9 n o$ & M(Papá 0 años) & $\mathbf{9 3 . 0}$ & IE Adecuada \\
5 & 22 & $F$ & $9 n o$ & M(Papá /Mamá 6 años) & $\mathbf{8 6 . 0}$ & IE Adecuada \\
6 & 18 & $F$ & 3ro & M(Papá 0 años) & $\mathbf{1 1 2 . 0}$ & IE Alta \\
7 & 18 & $F$ & 4 to & M(Papá 3 años) & $\mathbf{8 2 . 0}$ & IE Adecuada \\
8 & 20 & $M$ & 3ro & M(Papá 10 años) & $\mathbf{9 1 . 0}$ & IE Adecuada \\
9 & 20 & $M$ & 2do & M(Papá 8 años) & $\mathbf{1 0 0 . 0}$ & IE Adecuada \\
10 & 20 & $F$ & 3ro & M(Papá 0 años) & $\mathbf{7 3 . 0}$ & IE Adecuada \\
11 & 19 & $M$ & 5to & M(Mamá 12años) & $\mathbf{4 9 . 0}$ & IE Poca \\
12 & 20 & $F$ & 6to & M(Papá 3 años) & $\mathbf{8 0 . 0}$ & IE Adecuada \\
13 & 20 & $M$ & 3ro & M(Papá 5 años) & $\mathbf{6 8 . 0}$ & IE Poca \\
14 & 18 & $M$ & 2do & M(Papá 0 años) & $\mathbf{6 4 . 0}$ & IE Poca \\
15 & 18 & $F$ & 2do & M(Papá 3 años) & $\mathbf{7 7 . 0}$ & IE Adecuada \\
16 & 20 & $M$ & 5to & M(Papá 9 años) & $\mathbf{8 1 . 0}$ & IE Adecuada \\
17 & 21 & $F$ & 6to & M(Papá 10 años) & $\mathbf{7 3 . 0}$ & IE Adecuada \\
\hline
\end{tabular}




\begin{tabular}{|c|c|c|c|c|c|c|}
\hline $\begin{array}{c}\text { No. } \\
\text { Estudiantes }\end{array}$ & Edad & Sexo & Trimestre & Familia tipo & $\begin{array}{l}\text { Puntaje } \\
\text { Total }\end{array}$ & $\begin{array}{c}\text { Nivel de Inteligencia } \\
\text { Emocional }\end{array}$ \\
\hline 18 & 18 & $\mathrm{~F}$ & 4 to & M(Papá 2 años) & 120.0 & IE Alta \\
\hline 19 & 18 & M & 3 ro & M(Papá 6 años) & 76.0 & IE Adecuada \\
\hline 20 & 18 & $\mathrm{~F}$ & 3 ro & M(Papá 13 años) & 107.0 & IE Alta \\
\hline 21 & 19 & $\mathrm{~F}$ & 4 to & M(Papá 1 año) & 101.0 & IE Adecuada \\
\hline 22 & 19 & $\mathrm{~F}$ & 3 ro & M(Papá 3 años) & 72.0 & IE Adecuada \\
\hline 23 & 19 & $\mathrm{~F}$ & 6 to & M(Papá 2 años) & 98.0 & IE Adecuada \\
\hline 24 & 21 & M & 4 to & M(Papá 10 años) & 91.0 & IE Adecuada \\
\hline 25 & 19 & $\mathrm{~F}$ & 4 to & M(Papá 1 año) & 89.0 & IE Adecuada \\
\hline 26 & 24 & M & 2do & M(Papá 6 años) & 73.0 & IE Adecuada \\
\hline 27 & 18 & $\mathrm{~F}$ & 5to & M(Papá 0 años) & 88.0 & IE Adecuada \\
\hline 28 & 28 & M & $8 \mathrm{vo}$ & M(Papá 13 años) & 92.0 & IE Adecuada \\
\hline 29 & 20 & $\mathrm{~F}$ & 5 to & M(Papá 13 años) & 84.0 & IE Adecuada \\
\hline 30 & 20 & $\mathrm{~F}$ & $7 \mathrm{mo}$ & M(Papá 8 años) & 68.0 & IE Poca \\
\hline 31 & 18 & $\mathrm{~F}$ & 3ro & M(Papá 2 años) & 101.0 & IE Adecuada \\
\hline 32 & 18 & $\mathrm{~F}$ & 3ro & M(Papá 10 años) & 64.0 & IE Poca \\
\hline 33 & 18 & M & 3ro & M(Papá 13 años) & 101.0 & IE Adecuada \\
\hline 34 & 22 & $\mathrm{~F}$ & 5 to & M(Mamá 13años) & 71.0 & IE Adecuada \\
\hline 35 & 18 & $\mathrm{~F}$ & 3 ro & M(Papá 6 años) & 70.0 & IE Poca \\
\hline 36 & 19 & $\mathrm{~F}$ & 6 to & M(Papá 1 año) & 82.0 & IE Adecuada \\
\hline 37 & 19 & M & 2do & M(Mamá 10años) & 96.0 & IE Adecuada \\
\hline 38 & 19 & $\mathrm{~F}$ & 3 ro & M(Mamá 1 año) & 80.0 & IE Adecuada \\
\hline 39 & 23 & $M$ & 5 to & M(Papá 4 años) & 78.0 & IE Adecuada \\
\hline 40 & 19 & $\mathrm{~F}$ & 3ro & M(Papá 9 años) & 74.0 & IE Adecuada \\
\hline 41 & 23 & $M$ & $8 \mathrm{vo}$ & M(Mamá 10años) & 92.0 & IE Adecuada \\
\hline 42 & 20 & $M$ & 6 to & M(Papá 4 años) & 93.0 & IE Adecuada \\
\hline 43 & 21 & $\mathrm{~F}$ & 5to & M(Papá 9 años) & 96.0 & IE Adecuada \\
\hline 44 & 20 & $\mathrm{~F}$ & 2do & M(Papá 8 años) & 84.0 & IE Adecuada \\
\hline 45 & 22 & $\mathrm{~F}$ & 6to & M(Mamá 10años) & 82.0 & IE Adecuada \\
\hline 46 & 20 & $\mathrm{~F}$ & 3ro & M(Papá 11 años) & 95.0 & IE Adecuada \\
\hline 47 & 18 & $\mathrm{~F}$ & 2do & M(Papá 0 años) & 72.0 & IE Adecuada \\
\hline 48 & 18 & M & 4 to & M(Papá 4 años) & 100.0 & IE Adecuada \\
\hline
\end{tabular}




\begin{tabular}{|c|c|c|c|c|c|c|}
\hline $\begin{array}{c}\text { No. } \\
\text { Estudiantes }\end{array}$ & Edad & Sexo & Trimestre & Familia tipo & $\begin{array}{l}\text { Puntaje } \\
\text { Total }\end{array}$ & $\begin{array}{c}\text { Nivel de Inteligencia } \\
\text { Emocional }\end{array}$ \\
\hline 49 & 20 & $M$ & 2do & M(Papá 0 años) & 81.0 & IE Adecuada \\
\hline 50 & 24 & $\mathrm{~F}$ & $8 \mathrm{vo}$ & M(Papá 0 años) & 76.0 & IE Adecuada \\
\hline 51 & 20 & $\mathrm{~F}$ & $8 \mathrm{vo}$ & M(Papá 5 años) & 44.0 & IE Poca \\
\hline 52 & 19 & $\mathrm{~F}$ & 4 to & M(Papá 2 años) & 90.0 & IE Adecuada \\
\hline 53 & 20 & $\mathrm{~F}$ & $8 \mathrm{vo}$ & M(Papá 9 años) & 80.0 & IE Adecuada \\
\hline 54 & 18 & $\mathrm{~F}$ & 3ro & M(Papá 5 años) & 84.0 & IE Adecuada \\
\hline 55 & 18 & $\mathrm{~F}$ & 3 ro & M(Papá 1 año) & 82.0 & IE Adecuada \\
\hline 56 & 21 & M & 9no & M(Papá 0 años) & 98.0 & IE Adecuada \\
\hline 57 & 20 & M & 3ro & M(Papá 5 años) & 86.0 & IE Adecuada \\
\hline 58 & 26 & $M$ & 5 to & M(Papá 12 años) & 98.0 & IE Adecuada \\
\hline 59 & 21 & $\mathrm{~F}$ & 6to & M(Mamá 13años) & 72.0 & IE Adecuada \\
\hline 60 & 22 & $\mathrm{~F}$ & 9 no & M(Papá 13 años) & 81.0 & IE Adecuada \\
\hline 61 & 23 & $\mathrm{~F}$ & 8vo & M(Papá 13 años) & 69.0 & IE Poca \\
\hline 62 & 23 & $\mathrm{~F}$ & $8 \mathrm{vo}$ & M(Papá 5 años) & 93.0 & IE Adecuada \\
\hline 63 & 22 & M & $8 \mathrm{vo}$ & M(Papá 8 años) & 56.0 & IE Poca \\
\hline 64 & 23 & $\mathrm{~F}$ & $8 \mathrm{vo}$ & M(Papá 13 años) & 78.0 & IE Adecuada \\
\hline 65 & 23 & M & $8 \mathrm{vo}$ & M(Papá 0 meses) & 80.0 & IE Adecuada \\
\hline 66 & 23 & $\mathrm{~F}$ & $8 \mathrm{vo}$ & M(Papá 6 años) & 92.0 & IE Adecuada \\
\hline 67 & 19 & $\mathrm{~F}$ & 4 to & M(Papá 10 años) & 70.0 & IE Poca \\
\hline 68 & 18 & M & 4 to & M(Papá 2 años) & 60.0 & IE Poca \\
\hline 69 & 19 & $M$ & 4 to & M(Papá 12 años) & 65.0 & IE Poca \\
\hline 70 & 18 & $\mathrm{~F}$ & 4 to & M(Papá 2 años) & 97.0 & IE Adecuada \\
\hline 71 & 18 & $M$ & 3 ro & M(Papá 4 años) & 104.0 & IE Alta \\
\hline 72 & 20 & $\mathrm{~F}$ & 3ro & M(Papá 7 años) & 71.0 & IE Adecuada \\
\hline 73 & 28 & M & 9 no & M(Papá 5 años) & 109.0 & IE Alta \\
\hline 74 & 22 & $\mathrm{~F}$ & 9 no & M(Papá 8 años) & 91.0 & IE Adecuada \\
\hline 75 & 18 & $\mathrm{~F}$ & 2 do & M(Mamá 3años) & 79.0 & IE Adecuada \\
\hline 76 & 19 & $\mathrm{~F}$ & 4 to & M(Papá 12 años) & 92.0 & IE Adecuada \\
\hline 77 & 18 & $\mathrm{~F}$ & 3ro & M(Papá 4 años) & 79.0 & IE Adecuada \\
\hline 78 & 19 & $\mathrm{~F}$ & 4 to & M(Mamá 10años) & 114.0 & IE Alta \\
\hline
\end{tabular}




\begin{tabular}{ccccccc}
\hline $\begin{array}{c}\text { No. } \\
\text { Estudiantes }\end{array}$ & Edad & Sexo & Trimestre & Familia tipo & $\begin{array}{c}\text { Puntaje } \\
\text { Total }\end{array}$ & $\begin{array}{c}\text { Nivel de Inteligencia } \\
\text { Emocional }\end{array}$ \\
\hline 79 & 18 & $F$ & $3 r o$ & M(Papá 4 años) & $\mathbf{9 1 . 0}$ & IE Adecuada \\
80 & 20 & $F$ & 5 to & M(Mamá 10años) & $\mathbf{9 3 . 0}$ & IE Adecuada \\
81 & 20 & M & 3ro & M(Papá 5 años) & $\mathbf{9 4 . 0}$ & IE Adecuada \\
82 & 22 & $F$ & 3ro & M(Papá 0 meses) & $\mathbf{7 2 . 0}$ & IE Adecuada \\
83 & 18 & $F$ & 4 to & M(Mamá Omeses) & $\mathbf{9 1 . 0}$ & IE Adecuada \\
84 & 22 & M & 6to & M(Papá 8 años) & $\mathbf{9 8 . 0}$ & IE Adecuada \\
85 & 19 & M & 8vo & M(Mamá 5años) & $\mathbf{6 4 . 0}$ & IE Poca \\
86 & 20 & M & 3ro & M(Papá 7 años) & $\mathbf{9 1 . 0}$ & IE Adecuada \\
87 & 24 & M & 9 9no & M(Mamá 13años) & $\mathbf{7 3 . 0}$ & IE Adecuada \\
88 & 20 & $F$ & 3ro & M(Papá 4 años) & $\mathbf{1 2 0 . 0}$ & IE Alta \\
89 & 26 & M & 6to & M(Papá 7 años) & $\mathbf{1 0 3 . 0}$ & IE Adecuada \\
90 & 21 & $F$ & $9 n o$ & M(Papá 9 años) & $\mathbf{8 8 . 0}$ & IE Adecuada \\
\hline Media & 20 & & & & $\mathbf{8 4 . 8}$ & IE Adecuada \\
\hline DS & 2.3 & & & & $\mathbf{1 4 . 5}$ & \\
\hline
\end{tabular}

Fuente: Arraez K, Castro M (2018)

Se aprecia en la Tabla 2 que el $77.8 \%$ presentan IE adecuada, representando la capacidad de la mayoría para adaptarse y enfrentar las demandas y presiones del medio, esto conlleva a que IE apoya al individuo a tener resultados satisfactorios en su vida, tales como éxito en el trabajo, tolerancia al estrés en sus relaciones interpersonales, integración en trabajo de grupo, evidenciando que las habilidades basadas en IE una vez que se relacionan con la cotidianidad de la vida del estudiante, instauran modelos de competencias emocionales ávidos de ser transmitidos, ya que tienen su efecto en el ámbito familiar, la carrera profesional e inserción laboral.

Tabla 2. Distribución de los niveles de Inteligencia Emocional.

\begin{tabular}{cccc}
\hline Rangos de puntuaciones & Niveles de Inteligencia Emocional & f & $\%$ \\
\hline$>104$ & IE Alta & 7 & $7.8 \%$ \\
De 71 a 103 & IE Adecuada & 70 & $77.8 \%$ \\
$<70$ & IE Poca & 13 & $14.4 \%$ \\
& Totales & $\mathbf{9 0}$ & $\mathbf{1 0 0 . 0 \%}$ \\
\hline
\end{tabular}

Fuente: Arraez K, Castro M (2018) 


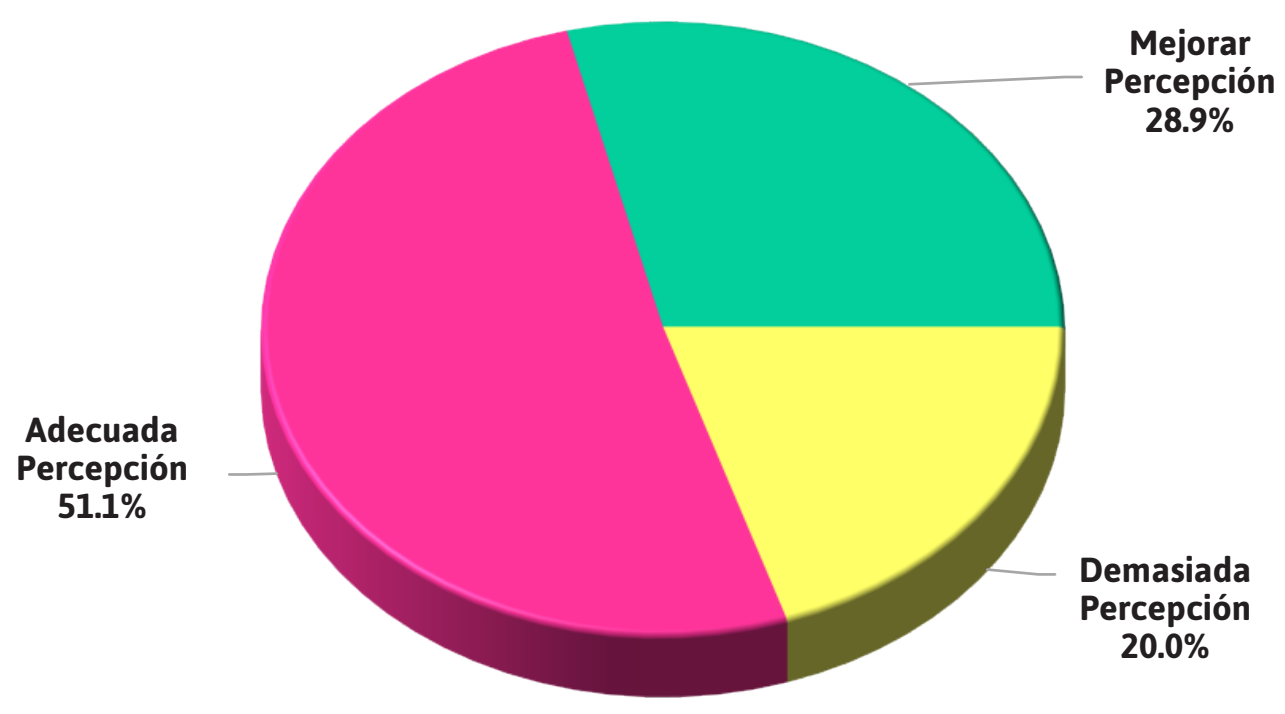

Gráfico 1. Distribución de los niveles de Percepción Emocional (Fuente: Arraez, y Castro, 2018).

De acuerdo a Fernández y Extremera (2005), "la percepción emocional es la habilidad que permite al individuo identificar y reconocer tanto los propios sentimientos como los de aquellos que le rodean". En el Gráfico 1 se expresa a porcentajes significativos relativos a la dimensión de la percepción, los cuales se diferencian así: el $51.1 \%$ adecuada percepción, $28.9 \%$ condición para mejorar la percepción y un $20 \%$ con demasiada percepción, esta habilidad implicaría la facultad para discriminar acertadamente la honestidad y sinceridad de las emociones expresadas por los demás. Es por ello que, las emociones una vez percibidas e reconocidas, deben ser expresadas y estimadas de manera apropiada para que no desencadenen emociones secundarias negativas en el individuo. Este resultado refleja que no todas las personas tienen la misma capacidad de percibir dichas emociones.

Por su parte, se observa en este Gráfico 2 que, el $55.6 \%$ refleja adecuada comprensión, el $15.6 \%$ manifiesta excelente comprensión, mientras que el $28.9 \%$, lo cual indica que los estudiantes con altas capacidades en este factor entienden perfectamente cómo influirá su comportamiento en otras personas, mejorando así sus relaciones sociales, haciendo más empático su proceder cotidiano, permitiéndole esta habilidad mayor control de sus emociones ponderando su racionalidad para la comprensión de su entorno. 


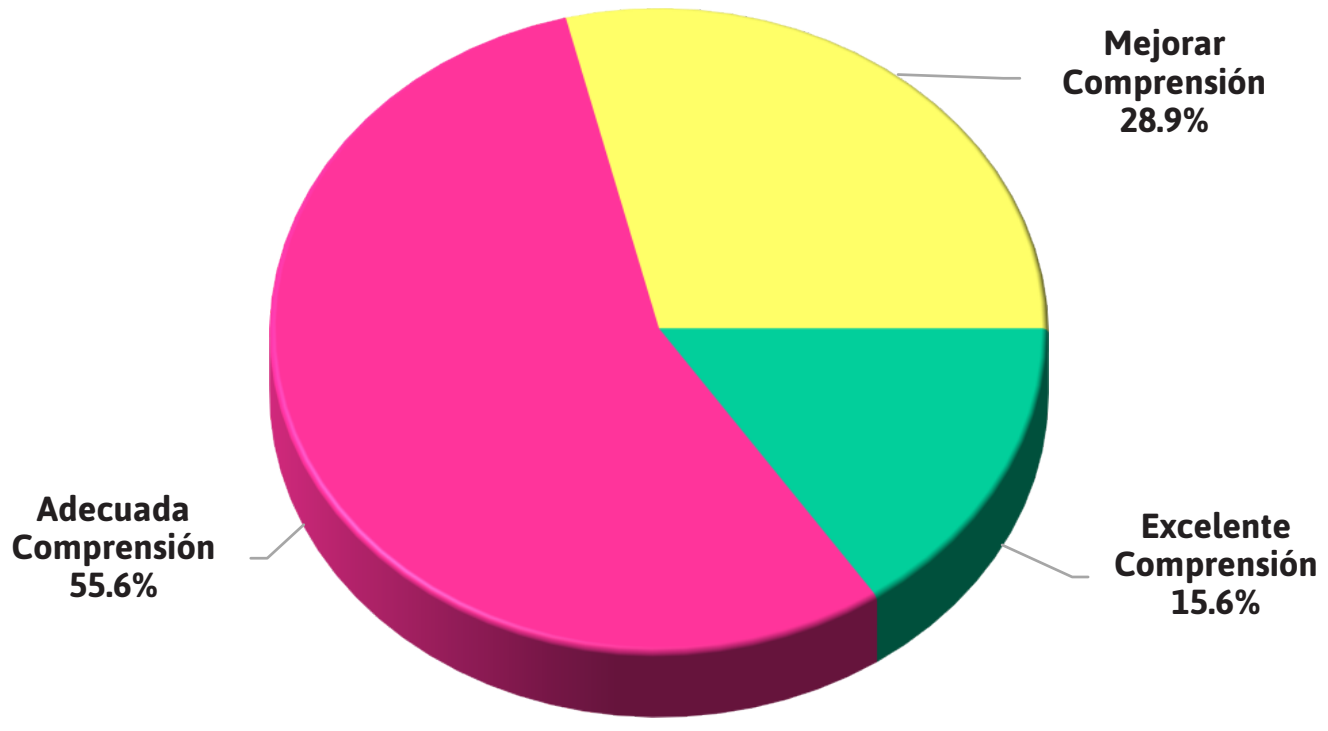

Gráfico 2. Distribución de los niveles de Comprensión emocional (Fuente: Arraez, y Castro, 2018).

Se determina en este Gráfico 3 la homogeneidad de la muestra a través de los siguientes porcentajes: $57.8 \%$ adecuada regulación, lo que indica que este grupo es capaz de regular su emociones, el $21.1 \%$ excelente regulación por lo que se hace evidente el manejo de su emociones y el $21.1 \%$ mejorar regulación, lo indica que debe esforzarse en mejorar, sin embargo no quiere decir que no se controlen. Es importante añadir que, a través de la regulación emocional se manejan las emociones de manera apropiada, teniendo estrategias de afrontamiento y capacidad de autogenerarse emociones positivas.

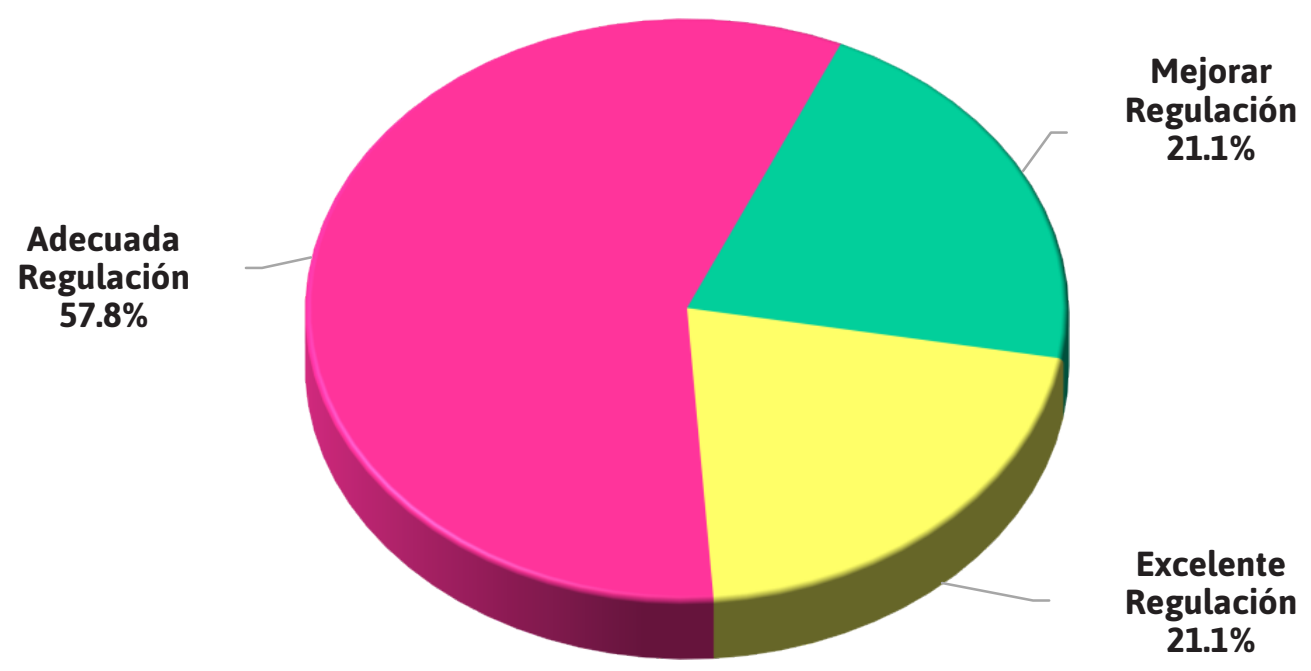

Gráfico 3. Distribución de los niveles de Regulación emocional. (Fuente: Arraez, y Castro, 2018). 
Se presentan pues puntuaciones medias como Adecuada percepción 27. 4\%, Adecuada comprensión 28. 2\% y Adecuada Regulación 29.2\%

\section{CONCLUSIONES}

La inteligencia emocional permite establecer niveles de salud mental, debido favorecimiento del soporte de la racionalidad y el equilibrio de las emociones ante las eventualidades del entorno, otorgando una interpretación más favorable del comportamiento, mayor conocimiento de sí mismo, así como una herramienta para aplicar las reacciones propias de huida o acercamiento ante las emociones que según convenga, implicando el mejoramiento personal del nivel de las relaciones intrapersonales e interpersonales, concediendo al individuo mayor ventaja de estas relaciones. Se cierra este estudio considerando que es preciso orientar a los estudiantes en cuanto a las creencias irracionales y desarrollar en ellos estrategias basadas en Inteligencia emocional para el equilibrio de sus emociones. Un camino académicos beneficioso son los conversatorios que permitan la interacción con los estudiantes y así poder determinar sus inquietudes.

\section{REFERENCIAS}

Abarca. (2007).Psicología del Niño en Edad Escolar. Disponible:https://books.google.co.ve/ books?id=PjnWBndwNJ8C\&pg=PA39\&dq=erick + e $r$ i c k s o n\& $h \mathrm{l}=$ e $s \& s a=X \& v$ e $d=$ 0 ahUKEwjhysjl 9 ZLbAhVHuVMKHc OahUKEwjhysj 19 Z LbAhVHuVMKHc4CsgQ6wEIJzAA\#v= $\quad$ onepage\&q=erick\%20 erickson\&f $=$ false

Boeree. (2006). Personality Theories. Disponible: http://www.social- psychology.de/do/pt_ erikson.pdf

Ceballos. (2015). Las inteligencias múltiples en educación infantil. Disponible en: https:// uvadoc.uva.es/bitstream/10324/14578/1/ TFG-G\%201368.pdf

Cherniss, C. y Goleman, D. (2005). Inteligencia Emocional. Editorial Kairos Barcelona España

Ellis, A., y Ibáñez, A. (1980). Razón y emoción en psicoterapia. Desclée de Brouwer
Goleman, D. (1985). La inteligencia emocional. Ediciones B. México, Vergara. S.A. de C.V

Hernández y Moreno. (2015). La ausencia de la figura paterna y su relación con la estructura de personalidad y la autoimagen de los adolescentes entre 18 a 20 años de edad. Universidad Arturo Michelena. San Diego, Estado Carabobo

Huerta, J. (2012). Inteligencia e Ideas Emocionales en estudiantes universitarios

Universidad Tecnológica de Huejotzingo. Puebla. México

Instituto Nacional de Estadísticas. INE. (2011). Censo Nacional de Población y Vivienda del año 2011. Recuperado de: http://www.ine.es/ inebase/index.html

Mazariegos, M. (2013). Relación entre las ideas irracionales y la comunicación disfuncional en la familia. INEB adscrito a la escuela normal de maestras de educación para el hogar de Quetzaltenango en Guatemala

Pereira, M. (2014). Estudio de la Inteligencia Emocional en el manejo de conflictos laborales de los empleados de la Oficina de Personal del Gobierno Bolivariano del Estado Carabobo. Universidad de Carabobo

Rosal, I., Dávila, M., Sánchez, S., Bermejo, M. (2016). La Inteligencia Emocional en estudiantes universitarios: Diferencias entre el Grado de Maestro en Educación primaria y los Grados en Ciencias". Universidad de Extremadura. España

Salovey, P., y Mayer, J.D., (1997). Emotional intelligence. Imagination, Cognition, and Personality. Editorial

Salovey, P., Mayer, J. D., Goldman, S. L., Turvey, C., Palfai, T. P. (1999). Emotional attention, clarity, and repair: exploring emotional intelligence using the Trait Meta-Mood Scale. J. W.Pennebaker (Ed.), Emotion, Disclosure, y Health

Segura, J., (2016). Inteligencia Emocional en estudiantes venezolanos de educación media y universitaria. Universidad Arturo Michelena. San Diego. Estado Carabobo

Valladares. (2008). La Familia. Una mirada desde la Psicología. Revista Médica Electrónica en Cienfuegos. Medisur

UNICEF. (2003). Declaración de América Latina y del Caribe. Familia Recuperado: https://elpais.com/diario/2003/10/25/ sociedad/106032802_850215.html 